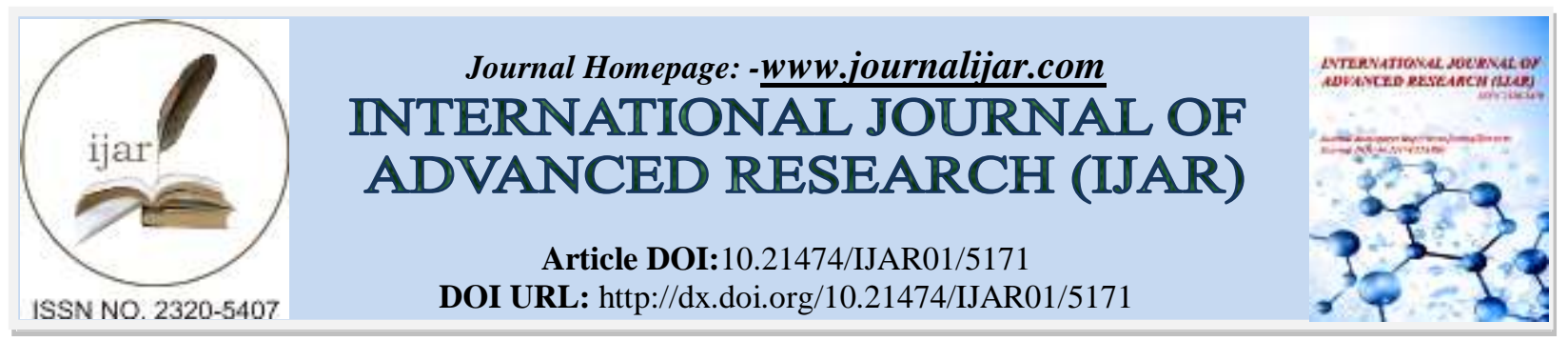

RESEARCH ARTICLE

\title{
STUDY OF SERUM VITAMIN D LEVELS IN PREECLAMPTIC WOMEN BELOW 35 YEARS IN A TERTIARY CARE CENTRE IN SOUTHERN ASSAM, INDIA.
}

\section{Aparajeeta Bora ${ }^{1}$, Mauchumi Saikia Pathak ${ }^{2}$, Arun Paul Choudhury ${ }^{3}$, Biswadeep Choudhury ${ }^{4}$ and Mainak Roy ${ }^{5}$.}

1. Department of Biochemistry,Silchar Medical College and Hospital, Silchar (Assam), India.

2. Professor and HOD, Department of Biochemistry, Silchar Medical College and Hospital, Silchar (Assam), India.

3. Associate professor,Department of Obstetrics \&Gynaecology, Silchar Medical College and Hospital, Silchar (Assam), India.

4. Associate Professor, Department of Biochemistry,Silchar Medical College and Hospital, Silchar (Assam), India.

5. Demonstrator, Department of Biochemistry, Silchar Medical College and Hospital, Silchar (Assam),India.

\section{Manuscript Info}

Manuscript History

Received: 14 June 2017

Final Accepted: 16 July 2017

Published: August 2017

Key words:-

Pre-eclampsia,vitamin D, pregnancy, supplementation.

\begin{abstract}
Pre-eclampsia,a hypertensive multisystem disorder is one of the major causes of maternal morbidity and mortality. The exact aetiology is still unknown, though numerous hypotheses have been put forward. Studies have found that low serum vitamin D level may be associated with development of pre-eclampsia.

The aim of this study was to find the association between serum vitamin D level and pre-eclampsia by comparing its levels in preeclamptic women with that of normal pregnant ladies. Our findings in this study indicate that pre-eclamptic women had a significantly lower serum vitamin $\mathrm{D}$ level than normal pregnant women $(\mathrm{p}<0.01)$ This study concludes that low serum vitamin D level may be a causal factor for the development of pre-eclampsia.
\end{abstract}

Copy Right, IJAR, 2017,. All rights reserved.

\section{Introduction:-}

Preeclampsia syndrome, a hypertensive multi-system disorder either alone or superimposed on chronic hypertension forms the deadly triad, with hemorrhage and infection. It has often been labeled the "disease of theories" due to the numerous hypotheses that drive research into this complex disease (Higgins JR and Brennecke $\mathrm{SP}, 1998)$. This complication of pregnancy is one of the most common causes of maternal morbidity [Duley L.(2009)].

Preeclampsia is characterized by high blood pressure $\geq 140 / 90$, oedema and proteinuria. It typically occurs sometime in the second or third trimesters — after 14 weeks of pregnancy — with symptoms like swelling of the hands and face, and sudden weight gain. In severe cases, symptoms include nausea, vomiting, vision changes, headache, belly pain,haemolysis, elevated liver enzymes, thrombocytopenia,pulmonary oedema and foetal growth restriction [Chesley LC(1978)] .It is a pregnancy-specific syndrome characterized by variable degrees of placental dysfunction and a maternal response featuring systemic inflammation(Evans KN, et al.2006). Studies suggest a failure of the trophoblastic invasion of the spiral arteries, leading to maladaptation of maternal spiral arterioles. This

Corresponding Author:-Aparajeeta Bora.

Address:-Department of Biochemistry,Silchar Medical College and Hospital, Silchar (Assam), India. 
is associated with an increased vascular resistance of the uterine artery and a decreased perfusion of the placenta. [Walker JJ(2000)].

The exact cause of preeclampsia is still unknown ,despite considerable research (Bringman $\mathrm{J}$,et.al.2006). The relationship between the aggravation of hypertension and the change in maternal serum level of various minerals during pregnancy have been shown in many clinical studies.

Vitamin D deficiency in pregnancy has been associated with an increased risk of pre-eclampsia(Kisters K,et.al.2000). It has been suggested to be a predisposing factor in the peripheral vascular phase modulation, which will result in inadequate placental development, decrease immunological tolerance for implantation and triggering of preeclampsia. The development of preeclampsia is linked to altered vitamin D metabolism, and might be a result from placental dysfunction (Fischer D,et.al.2007).

Research suggests intake of supplements, mainly vitamin D may help in the reduction of incidence of pre-eclampsia especially in a population of a developing country like India where the nutrition is poor. Hence,focus should be given more on prevention measures to manage preeclampsia. The present study aimed at measuring the serum vitamin D levels in pre-eclamptic women and comparing the same with that of normal pregnant women.

\section{Materials and Methods:-}

The present case-control study was conducted for a period of 1 year, among admitted pregnant ladies in the ward of O\&G . 100 pregnant women, primigravida ( $>20$ weeks of gestation) below 35 years with preeclampsia were included in the case group. Serum $25(\mathrm{OH}) \mathrm{D}$ levels of the case group were compared to that of 100 normotensive healthy pregnant women in the control group using Access 2 Immunoassay Systems, by the principle of chemiluminescence in the clinical laboratory .Statistical evaluation and correlation of findings was done using suitable statistical tools. All $p$ values $<0.05$ was considered as statistically significant. The data obtained were tabulated and entered in MS Excel Worksheet. Data were expressed as mean \pm SD. Independent $t$-test was applied for parametric variability considering $\mathrm{p}<0.05$ to be statistically significant. Data analysis was done by Graph Pad Instat (Version 3.10)

\section{Results:-}

The study is a observational hospital-based case control study of 100 preeclamptic (cases) and 100 normotensive pregnant (controls) of $<35$ years of age conducted in indoor ward of O\&G during July 2015 - July 2016.The study subjects were investigated for and results have been analysed by using unpaired student's test which is two tailed.

Table 1:-Showing mean serum vitamin D level of cases and controls along with SD,SEM and $\mathrm{p}$ value

\begin{tabular}{|l|l|l|l|l|l|}
\hline & Mean & SD & SEM & $95 \%$ CI for Mean & p-value \\
\hline Cases & 11.9 & 4.20 & 0.42 & {$[11.07,12.74]$} & $<0.01$ \\
\hline Controls & 21.97 & 3.28 & 0.33 & {$[21.32,22.63]$} & \\
\hline
\end{tabular}

The mean blood level of serum $25(\mathrm{OH})$ Cholecalciferol level in preeclampsia cases in this study was found to be [11.9 \pm 4.2$] \mathrm{ng} / \mathrm{ml}$ and $[21.9 \pm 3.28] \mathrm{ng} / \mathrm{ml}$ in controls . In the Unpaired t test between case and control groups, the two-tailed $\mathrm{P}$ value is $<0.01$ which is considered significant. 


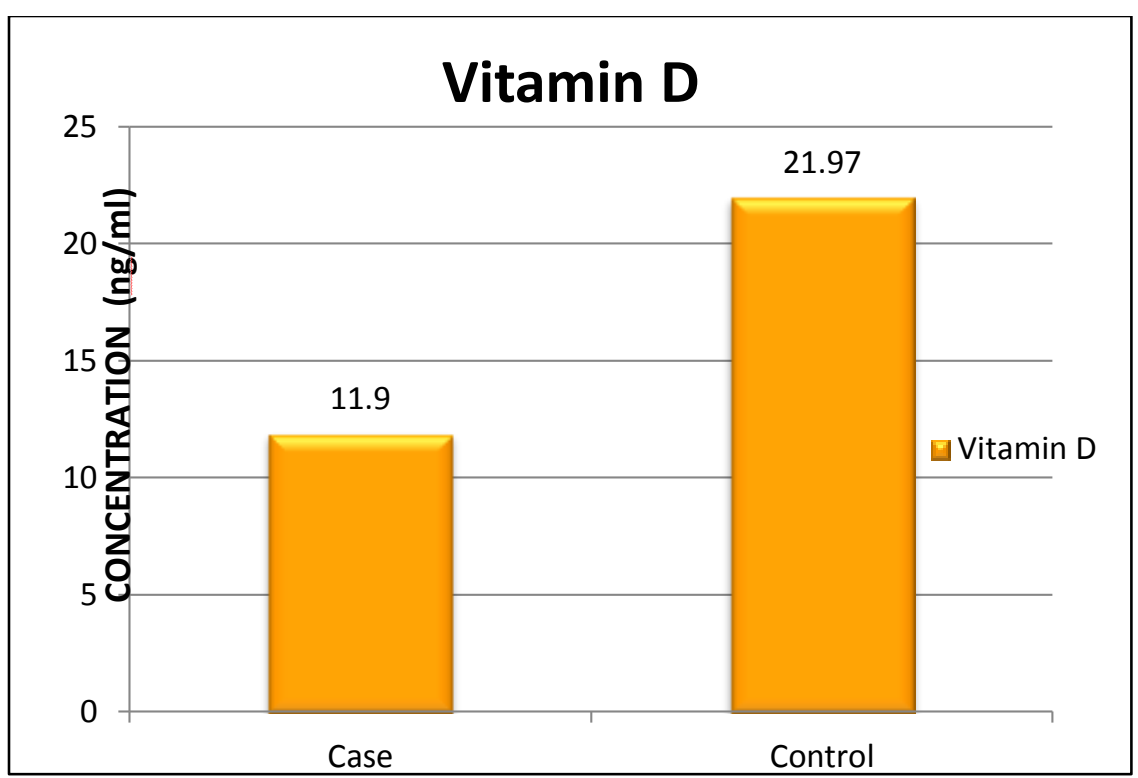

Figure1:- Showing mean value of serum vitamin D level in the study groups.

Table 2:- Distribution of study groups based on vitamin D status

\begin{tabular}{|l|l|l|l|}
\hline VITAMIN D STATUS & CASE & CONTROL & TOTAL \\
\hline DEFICIENCY $(<20 \mathrm{ng} / \mathrm{ml})$ & 98 & 24 & 122 \\
\cline { 2 - 4 } & $98 \%$ & $24 \%$ & $61 \%$ \\
\hline \multirow{2}{*}{ INSUFFICIENCY(20-30ng/ml) } & 2 & 75 & 77 \\
\cline { 2 - 4 } & $2 \%$ & $75 \%$ & $38.5 \%$ \\
\hline \multirow{2}{*}{ SUFFICIENCY $(30-100 \mathrm{ng} / \mathrm{ml})$} & 0 & 1 & 1 \\
\cline { 2 - 4 } & & $1 \%$ & $0.5 \%$ \\
\hline
\end{tabular}

The above table shows that $98 \%$ of cases were vitamin D deficient i.e. $(<20 \mathrm{ng} / \mathrm{ml})$ whereas in the control group $75 \%$ were found to be insufficient. Minimum sufficiency level 1\% was found in the control group.

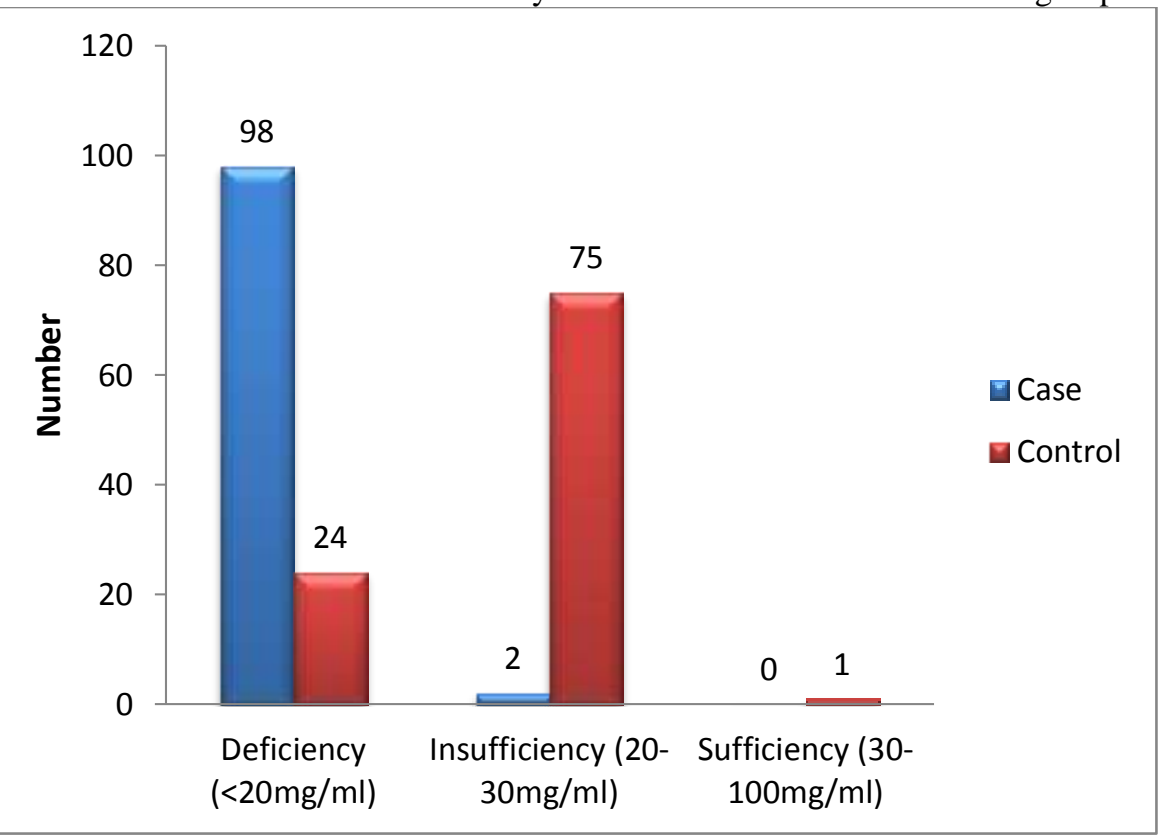

Figure 2:- Distribution of study groups based on vitamin D status 
Table 3:-Literacy,Community and economic status wise analysis of vitamin D levels in cases.

\begin{tabular}{|l|l|l|l|l|l|l|}
\hline LITERACY & Community & \multicolumn{2}{l|}{ Economic status } \\
\hline MEAN & Literate & Illiterate & Hindu & Muslim & BPL & NBPL \\
\cline { 2 - 7 } $\begin{array}{l}\text { VEVEL } \\
(\mathrm{ng} / \mathrm{ml})\end{array}$ & 12.05 & 11.79 & 12.99 & 11.71 & 11.6 & 12.1 \\
\hline
\end{tabular}

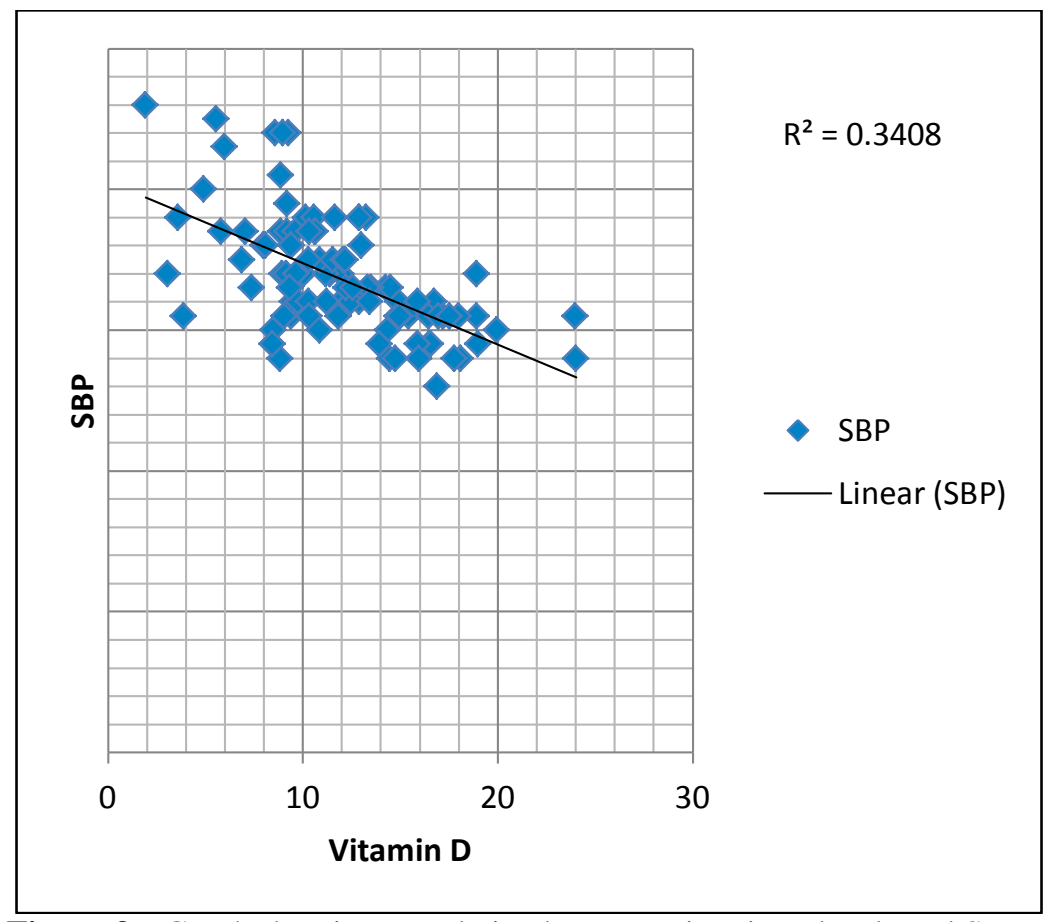

Figure 3:- Graph showing correlation between vitamin D levels and S.B.P. a)Pearson's correlation co-efficient $(r)=-0.548$, Coefficient of determination $\left(r^{2}\right)=0.34$.

The two-tailed $P$ value $=0.00$,

which indicates a statistically significant $(\mathrm{p}<0.05)$ negative correlation between vitamin $\mathrm{D}$ and systolic B.P. level.

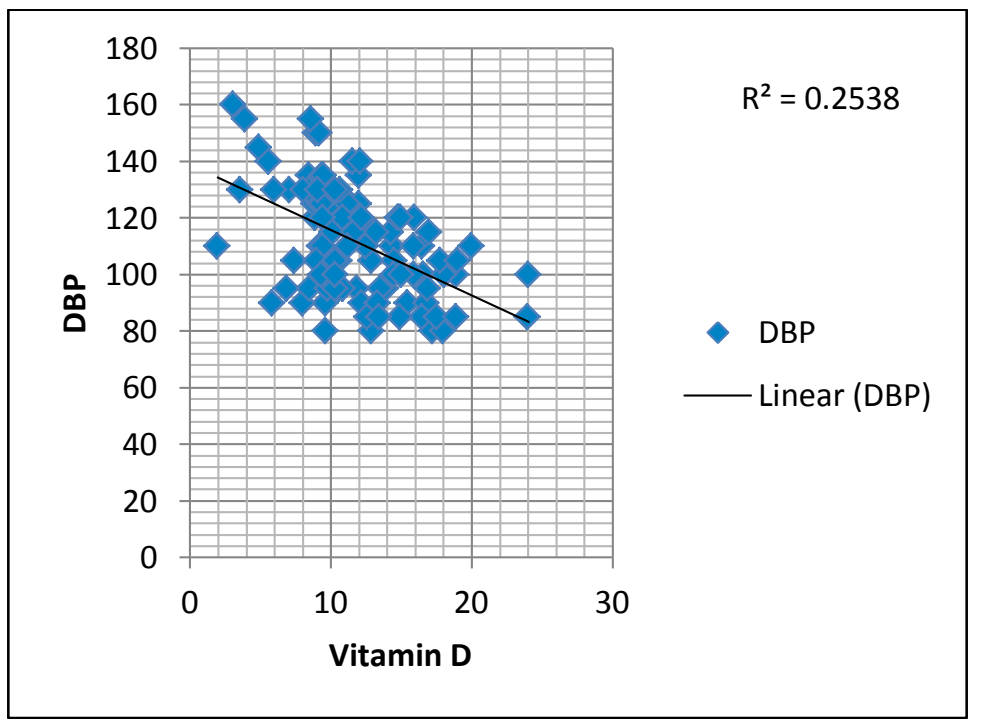

Figure 4:- Graph showing correlation between vitamin D levels and D.B.P. 
b) Pearson's correlation co-efficient $(r)=-0.504$ Coefficient of determination $\left(r^{2}\right)=0.253$

$P$ value $=\mathbf{0 . 0 0}$,

which indicates a statistically significant $(\mathrm{p}<0.05)$ negative correlation between vitamin $\mathrm{D}$ and diastolic B.P. level.

\section{Discussion:-}

In the present study,the mean blood level of serum $25(\mathrm{OH})$ Cholecalciferol in preeclampsia cases was found to be [11.1 \pm 4.2$] \mathrm{ng} / \mathrm{ml}$ and in age-matched controls, the mean level was $[21.9 \pm 3.28] \mathrm{ng} / \mathrm{ml}$. In the Unpaired t test between case and control groups, the two-tailed $\mathrm{P}$ value is $<0.01$ which is considered significant.

In the latest studies, vitamin D insufficiency and deficiency are diagnosed at levels of $<32 \mathrm{ng} / \mathrm{ml}$ and $<20 \mathrm{ng} / \mathrm{ml} 25$ $\mathrm{OH}-\mathrm{D}$, respectively.

In a meta-analysis of this issue, Tabesh et al. (Tabesh M, et. al.2013) established the association of low 25(OH)D level and preeclampsia.Studies with similar results are Hyppönen et al (Hypponen E,et.al.2014) which have shown association between risk of preeclampsia and low vitamin D levels.

Nassar et al (2011), a systematic review aimed at assessing normal levels of vitamin D in early pregnancy and its relation to subsequent pregnancy outcomes, no clear definition of vitamin D deficiency in pregnancy was obtained. They found insufficient evidence to suggest an association between low levels of vitamin D in early pregnancy and adverse pregnancy outcomes. (Nassar N,et.al.2011).

Several studies provided evidence for a dose-dependent association, with the recent trial by Hollis and Wagner (Hollis BW and Wagner CL,2013) providing the most notable example. Vitamin D is a promising candidate for preeclampsia prevention, and there is an urgent need for well-controlled randomised trials to test its effectiveness and safety.

In a relatively large prospective study performed in Norway by Haugen et al., the risk of PE was found to be decreased by $27 \%$ in patients who received vitamin D in a dose of 400-600 IU/daily through supplemental treatment. In addition, vitamin D support in the early weeks of pregnancy was shown to decrease the risk of encountering preeclampsia during the advanced phases of pregnancy. (Haugen M,et.al.2009)

In our study we found $98 \%$ cases and $24 \%$ controls were suffering from vitamin D deficiency. i.e. $(<20 \mathrm{ng} / \mathrm{ml})$. We can see a higher percentage of vitamin D deficiency in the case group compared to healthy controls. We are in accordance with several reports documenting a higher than normal prevalence of vitamin D deficiency in the preeclamptic population.

Also, in our study we found mean vitamin D levels in literate women were more compared to that of illiterate women.(P value $<0.01)$ Data in Muslim women have shown lower levels than Hindu women.BPL card holders were found to be of low mean vitamin $\mathrm{D}$ level.

Studies found low socioeconomic status as a risk factor for preeclampsia. (Bassir M.,et.al.2001)

As early as the 1950s, researchers described associations between measures of socioeconomic status and hypertension during pregnancy (Silva LM,et.al.2008). In 1955 Nelson studied maternal social class as measured by the husband's occupation in relation to the incidence of pre-eclampsia, and found no association [ Nelson TR(1955)] . In contrast, Davies et al., (Davies AM,et.al.1970) and, more recently, Haelterman et al. (Haelterman E,et.al.2003)found that, relative to women with a higher educational level, those with a low educational level had a higher risk of pre-eclampsia. Studies found women practising religious customs (burkhas) and other clothings have increased risk of vitamin D deficiency [Khadrawi I.(2013)].

In the present study, systolic and diastolic B.P. were higher in preeclamptic cases with low vitamin D levels. This is similar to findings in other studies, as the one done by M.Bakacak and colleagues, where systolic and diastolic blood pressures were significantly higher in the eclamptic and preeclamptic patient groups compared to the healthy pregnant women $(\mathrm{P}<0.001)($ Bakacak M,et.al.2015). 
PEARSON'S correlation was derived between vitamin D levels with systolic and diastolic blood pressures. A significant negative correlation was established between the parameters which infers that as the vitamin D level lowers, increase in blood pressure occur.

\section{Conclusions:-}

Diagnosed cases of vitamin D deficiency have shown significant association with development of preeclampsia. Thus, vitamin D may be considered to be an important factor in development of preeclampsia and may have therapeutic implications as cautious vitamin D supplementation may improve symptoms of preeclampsia and halt development of complications .

\section{Acknowledgement:-}

The authors are thankful to the Department of Biotechnology (DBT), Ministry of Science and Technology, Government of India for the financial support.

\section{References:-}

1. Higgins JR,BrenneckeSP.Pre-eclampsia-still a disease of theories ?CurrOpinObstet Gynecol.1998;10(2):129-33.

2. DuleyL.The global impact of pre-eclampsia and eclampsia. Seminars in Perinatology,2009 Jun;33(3):130-137.

3. Chesley LC. Hypertensive disorders in pregnancy. New York, NY: Appleton-Century- Crofts; 1978.

4. Evans KN et al. Effects of 25-hydroxyvitamin D3 and 1,25-dihydroxyvitamin D3 on cytokine production by human decidual cells. Biology of Reproduction. 2006; 75:816-822.

5. Walker JJ. Pre-eclampsia. Lancet 2000; 356: 1260-5.

6. Bringman J, Gibbs C, Ahokas R. Differences in serum calcium and magnesium between gravidas with severe preeclampsia and normotensive controls. Am J ObstetGynaecol. 2006; 195:148.

7. Kisters K, Barenbrock M, Louwen F, Hausberg M, Rahn K H, Kosch M. Membrane, intracellular and plasma magnesium and calcium concentrations in pre-eclampsia. Am J Hypertens 2000; 13: 765-9.

8. Wei SQ,AudibertF,HidiroglouN,SarafinK,JulienP,WuY,LuoZC,Fraser WD. Longitudinal vitamin D status in pregnancy and the risk of pre-eclampsia.BCOG: an international journal of obstetrics and gynaecology 2012;119(7):832-9.

9. Fischer D,SchroerA,LuddersD,CordesT,BuckerB,ReichrathJ,FriedrichM.Metabolism of vitamin D3 in the placental tissue of normal and preeclampsia complicated pregnancies and premature births.ClinExpObstet Gynecol.2007;34:804.

10. TabeshM,Salehi-AbargoueiA,EsmaillzadehA.Maternal vitamin D status and risk of pre-eclampsia:a systematic review and meta-analysis.JClinEndocrinol Metab.2013;98:3165-73.

11. HypponenE,CavadinoA,WilliamsD,etal.Vitamin D and Pre-eclampsia:Original data, systematic review and Metaanalysis.AnnNutr Metab.2014;63:331-340.

12. NassarN,HalliganGH,RobertsCL,MorrisJM,AshtonAW.Systematic review of first-trimester vitamin D normative levels and outcomes of pregnancy.American journal of obstetrics and gynecology.2011 Sep30;205(3):208-e1.

13. Hollis BW,WagnerCL:Vitamin D and pregnancy:skeletaleffects,nonskeletaleffects, and birth outcomes. Calcif Tissue Int 2013;92:128-139.

14. Haugen M,BrantsaeterAL,TrogstadL,Alexander J, Roth C,MagnusP,MeltzerHM.Vitamin D supplementation and reduced risk of preeclampsia in nulliparous women.Epidemiology.2009 Sep;20(5):720-6.

15. .Bassir M.,LaborieS.,Lapillonne.A.,Claris O.,Chappuis, M.C.,and Salle B.L.Vitamin D deficiency in Iranian mothers and their neonates:a pilot study.ActaPaediatr. 2001;90:577-579

16. Silva LM,CoolmanM,SteegersEA,JaddoeVW,MollHA,HofmanA,MackenbachJP,Raat H.J Hypertens.Low socioeconomic status is a risk factor for preeclampsia :the Generation R study.2008 Jun;26(6):1200-8.

17. Nelson TR.A clinical study of pre-eclampsia.I.JObstetGynaecol Br Emp 1955;62:48-57.

18. Davies AM,CzaczkesJW,SadovskyE,PrywesR,WeiskopfP,SterkVV.Toxemia of pregnancy in Jerusalem.I.Epidemiological studies of a total community.Isr J Med Sci 1970;6:253-266

19. HaeltermanE,QvistR,BarlowP,AlexanderS.Social deprivation and poor access to care as risk factors for severe preeclampsia.Eur J ObstetGynecolReprodBiol 2003;111:25-32.

20. Khadrawi I. Vitamin D Supplementation Practices during Pregnancy and Infancy among a Sample of Muslim Women in Ireland and Saudi Arabia.2013

21. Bakacak M, Serin S, Ercan O, Köstü B, Avci F, Kılınç M, Kıran H, Kiran G. Comparison of vitamin D levels in cases with preeclampsia, eclampsia and healthy pregnant women. International journal of clinical and experimental medicine. 2015;8(9):16280. 